\title{
Das Protektorat Böhmen und Mähren und seine Rechtsordnung (1939-1945)
}

The Protectorate of Bohemia and Moravia and its Legal System (1939-1945)

After the establishment of the Protectorate of Bohemia and Moravia, a dual legal system was implemented on the territory of Bohemia and Moravia - Protectorate law (autonomous) and German law (imperial). Its application was usually based on the citizenship of the subjects of the legal relations. Along with the introduction of legal dualism, a German judiciary was also established, parallel to the comprehensive German occupation administrative apparatus led by the Reich Protector. Throughout the existence of the Protectorate, German officials kept intervening into the autonomous law, therefore the often proclaimed 'autonomy' was gradually fading away. In the contribution, the collections of publications in which provisions of the imperial law as well as the autonomous law were published, will be introduced.

Keywords: autonomous law - imperial law - legal system - Protectorate of Bohemia and Moravia publication of legal regulations

\section{Einführung}

Als Ende September 1938 das Münchner Abkommen zwischen den vier europäischen Großmächten geschlossen wurde und die Tschechoslowakei auf Grund des Abkommens gezwungen war, ihre umfangreichen Grenzgebiete an das Deutsche Reich abzutreten, versicherten die deutschen Vertreter der Weltöffentlichkeit, "das tschechische Problem" sei damit gelöst und das Deutsche Reich werde keine weiteren Gebietsansprüche gegenüber der Tschechoslowakei erheben. Wie sich jedoch gleich im Frühjahr des kommenden Jahres zeigte, hielt sich das Deutsche Reich nicht an diese Ankündigung.

Bei den Verhandlungen mit Adolf Hitler in der Nacht vom 14. auf 15. März 1939 in der Reichskanzlei war der Präsident der zweiten Tschechoslowakischen Republik Dr. Emil Hácha gezwungen, eine Erklärung zu unterzeichnen, in welcher er das Schicksal des tschechischen Vol- kes und des Staates in die Hände des Führers des deutschen Volkes legte. Für deutsche Propagandazwecke war dieser Akt von großer Bedeutung, er sollte den Schein der Freiwilligkeit und damit auch der Legalität erwecken, was einerseits für künftige staatsrechtliche Begründung des Protektorats und andererseits für die Rechtfertigung der Besetzung sehr wichtig war. ${ }^{1}$

\footnotetext{
${ }^{1}$ Zur Errichtung des Protektorats im deutschsprachigem Schrifttum z.B. RILL, Böhmen und Mähren 904ff. oder BRANDES, Die Tschechen.
} 


\section{Der Erlass des Führers} und Reichskanzlers über das Protektorat Böhmen und Mähren

Die Rechtsgrundlage des Protektorats war Hitlers Erlass² vom 16. März 1939, der im Rundfunk verkündet, in fast allen tschechischen Tageszeitungen abgedruckt und auch in einigen amtlichen Verkündungsblättern ${ }^{3}$ verlautbart wurde. Dieser Erlass bestand aus einer Präambel und 13 Artikeln und war sehr unklar und unkonkret formuliert, sodass seine Bestimmungen eine Doppelauslegung zuließen, was von den Besatzern auch ausgenutzt wurde. Im Laufe des Jahres 1939 wurde der Führererlass über das Protektorat Böhmen und Mähren noch durch zwei Rechtsvorschriften verfassungsrechtlichen Charakters ergänzt. ${ }^{4}$ Die tschechoslowakische Verfassung von 1920 wurde nicht direkt aufgehoben, jedoch traten ihre dem Sinne der Übernahme des Schutzes durch das Deutsche Reich widersprechenden Bestimmungen außer Kraft. Welche Vorschriften außer Kraft zu treten hatten, wurde vom Reichsprotektor festgesetzt. ${ }^{5}$

Die Präambel als nicht normativer Teil des Erlasses wies direkt auf die Zugehörigkeit der böhmisch-mährischen Länder zum Reich hin

\footnotetext{
${ }^{2}$ Zur Entstehungsgeschichte des Erlasses des Führers und Reichskanzlers vom 16.3.1939 s. ausführlich MARŠÁLEK, Pod ochranou hákového kř́že 16-25.

${ }^{3}$ In deutscher Fassung in der Nr. 47 des Reichsgesetzblattes (dRGBl. I, S. 485) vom 16. 3. 1939, in deutscher und tschechischer Fassung in der Nummer 2 des Verordnungsblattes für Böhmen und Mähren (VB1., S. 7) vom 21.3. 1939 und am 17.3. 1939 im 18. Stück unter der Nr. 75 der Sammlung der Gesetze und Verordnungen.

${ }^{4}$ Es handelte sich um die Durchführungsverordnung vom 22. 3. 1939 (dRGBl. I, S. 542) und die Verordnung über den Aufbau der Verwaltung und die Deutsche Sicherheitspolizei im Protektorat Böhmen und Mähren vom 1. 9. 1939 (dRGBl. I, S. 1681).

${ }^{5}$ Verordnung über das Rechtsetzungsrecht im Protektorat Böhmen und Mähren vom 7.6.1939 (dRGBl. I, S. 1039).
}

und rekapitulierte die historische Entwicklung zwischen den böhmischen Ländern und dem Reich so, wie diese von den deutschen Vertretern gesehen wurde. ${ }^{6}$ Das neu errichtete und als „Protektorat Böhmen und Mähren“ bezeichnete Gebilde genoss den Schutz des Deutschen Reiches und Hitlers Erlass deutete die Existenz der Autonomie dieses geschaffenen Staatengebildes an. Das Protektorat Böhmen und Mähren wurde also für autonom erklärt und sollte das Recht haben, sich selbst zu verwalten, wobei seine Hoheitsrechte im Einklang mit den politischen, militärischen und wirtschaftlichen Belangen des Reiches ausgeübt werden mussten. Diese seitens der Deutschen oft betonte Autonomie galt jedoch nur auf dem Papier und konnte von den Nationalsozialisten jederzeit verletzt, beschränkt oder völlig liquidiert werden (s. Art. 5 und 11 des Erlasses), was darin zum Ausdruck gebracht wurde, dass das Reich Rechtsvorschriften mit Geltung für das Protektorat erlassen konnte. Nach und nach griffen die Nationalsozialisten immer mehr in diese "Autonomie" ein, bis sie diese in einigen Gebieten völlig abgeschafft hatten. Das Protektorat Böhmen und Mähren war also de facto ein Vasallenstaat und kein Protektorat in dem Sinne, in welchem es vom Völkerrecht verstanden wird. ${ }^{7}$

Die volksdeutschen Bewohner des Protektorates wurden deutsche Staatsangehörige und nach den Vorschriften des Reichsbürgergesetzes vom 15. September 1935 (RGB1. I, S. 1146) auch Reichsbürger. Sie waren aus der Zuständigkeit der autonomen (tschechischen) Gerichtsbarkeit ausgenommen und unterstanden nur der deutschen Gerichtsbarkeit. ${ }^{8}$ Nach dem Erlass Hitlers

${ }^{6}$ Zur Beziehung des tschechischen Staates und des Reiches und zu ihrer Auffassung in der Zeit des Protektorats s. ausführlich: TAUCHEN, Vztah českého státu 106-120.

${ }^{7}$ MARŠÁLEK, Pod ochranou hákového kř́íže 23.

${ }^{8}$ Diese Ausnahme wurde aufgrund folgender Verordnungen des Reichsjustizministers vom 14.4. 1939 durchgeführt: Verordnung über die deutsche Ge- 
wurden die übrigen Bewohner von Böhmen und Mähren (Tschechen) Staatsangehörige des Protektorates, was eine Äußerung der gewährten Autonomie war. Die Staatsangehörigen des Protektorats waren den deutschen Staatsangehörigen jedoch nicht gleichgestellt, die Tschechen verfügten über keine politischen Rechte und waren in einigen Rechtsgebieten (z.B. im Arbeits- und Sozialrecht) eklatant benachteiligt.

\section{Die Geltung des autonomen Rechts und des Reichsrechts}

Auf dem Gebiet des Protektorats galten zwei Rechtsysteme: das sog. autonome Recht, d.h. die aus der Zeit der Ersten und Zweiten Tschechoslowakischen Republik übernommenen und die neu von den autonomen Organen erlassenen Rechtsvorschriften, und das Reichsrecht, bzw. einige seiner Rechtsnormen.

$\mathrm{Ob}$ das autonome oder das Reichsrecht angewendet wurde, war üblicherweise davon abhängig, welche Staatsangehörigkeit das Rechtsubjekt besaß. Für die Protektoratsangehörigen war das rezipierte bisherige tschechoslowakische Recht einschlägig, jedoch musste dieses in den neuen staatsrechtlichen Verhältnissen im Geiste der Ideologie des nationalsozialistischen Großdeutschen Reiches ausgelegt werden. ${ }^{9}$ Die deutschen Staatsangehörigen, die ihren Wohnsitz im Protektorat hatten, besaßen

richtsbarkeit im Protektorat Böhmen und Mähren (dRGBl. I, S. 752), Verordnung über die Ausübung der Strafgerichtsbarkeit im Protektorat Böhmen und Mähren (dRGBl. I, S. 754) und Verordnung über die Ausübung der bürgerlichen Rechtspflege im Protektorat Böhmen und Mähren (dRGBl. I, S. 759).

${ }^{9}$ KRIESER, Die deutsche Gerichtsbarkeit 1745; ein Beispiel für die unterschiedliche Auslegung in der Zeit der Ersten Tschechoslowakischen Republik und im Protektorat stellt das Gesetz zum Schutze der Republik von 1923 dar. zusätzlich auch die Rechte der tschechischen Staatsangehörigen des Protektorats.

Weder für die Staatsangehörigen des Protektorats noch für deutsche Staatsangehörige galt das Reichsrecht als ein Ganzes, ein einheitliches Recht in dem gesamten Gebiet des Großdeutschen Reiches gab es nämlich nicht. ${ }^{10}$ Auf die Rechtsverhältnisse deutscher Staatsangehöriger war im Protektorat ihr Heimatrecht (also das im alten Reichsgebiet, in der Ostmark oder im Reichsgau Sudetenland geltende deutsche Recht) anzuwenden, soweit dieses Recht die Gesetze des Heimatstaates für anwendbar erklärte. Demgemäß galt das Heimatrecht insbesondere auf dem Gebiet des Personen-, Familien- und Erbrechts. Die Rechtsverhältnisse von Personen, welche die deutsche Staatsangehörigkeit im Zusammenhang mit der Errichtung des Protektorats erworben hatten, wurden nach dem deutschen Recht, wie es im Reichsgau Sudetenland galt, beurteilt, soweit dieses Recht die Gesetze des Heimatstaates für anwendbar erklärte. ${ }^{11}$

\section{Das autonome Recht}

Die erste Gruppe des auf dem Protektoratsgebiet geltenden Rechts stellt das sog. autonome Recht dar. Nach dem Ermächtigungsgesetz vom

\footnotetext{
${ }^{10}$ Verordnung über die Anwendung deutschen Rechts auf deutsche Staatsangehörige im Protektorat Böhmen und Mähren vom 20.7.1939 (dRGBl. I, S. 1309); aus dem Schrifttum dazu z.B. ŠTAJGR, Druhé nařízení 177-181.

${ }^{11}$ Aus den sich auf die allgemeine Rechtsentwicklung im Protektorat beziehenden Werken s. vor allem MARŠÁLEK, Pod ochranou hákového kříže 109-134, DERS., Protektorát Čechy a Morava 89-108; JANEČKOVÁ, Státoprávní uspořádání 108-152; aus dem deutschsprachigen Schrifttum s. vor allem SCHELLE, TAUCHEN, Recht und Verwaltung 101-120; KRIESER, Das neue Recht.
} 
15. Dezember 1938 (Nr. 330/1938 Slg.) $)^{12}$ war der Präsident ermächtigt, auf einstimmigen Vorschlag der Regierung Dekrete zur Verfassungsänderung zu erlassen. Die Regierung konnte zwei Jahre lang mit Zustimmung des Präsidenten Verordnungen mit Gesetzeskraft erlassen. Nach dem Ablauf der Ermächtigung wurde diese durch den Reichsprotektor Konstantin von Neurath im Dezember 1940 bis auf Weiteres verlängert, was die Abhängigkeit der tschechischen Regierung von den deutschen Reichsorganen noch betonte. ${ }^{13}$

Auch in der Zeit des Protektorats oblag die gesetzgebende Gewalt dem Staatspräsidenten und der Regierung. Die Nationalversammlung wurde vom Staatspräsidenten Hácha am 21. März 1939 aufgelöst. Die Ermächtigung zum Erlass von Verfassungsdekreten wurde von dem Staatspräsidenten Hácha nur ein einziges Mal, und zwar im März 1940, genutzt. ${ }^{14}$ Zum ausschließlichen Gesetzgeber wurde also die Protektoratsregierung, welche die Regierungsverordnungen erließ. Vom Beginn des Protektorats hatten also deutsche Organe die "autonome“ Normbildung des Protektorats wesentlich beeinflusst.

In das autonome Recht konnte auch der Reichsprotektor durch seine legislative Tätigkeit eingreifen; er war befugt, sich über alle Maßnahmen der Regierung des Protektorates unterrichten zu lassen und ihr Ratschläge zu erteilen. Er konnte gegen Maßnahmen, die das Reich zu schädigen geeignet waren, Einspruch einlegen

\footnotetext{
${ }^{12}$ Dazu ausführlich TAUCHEN, Ermächtigungsgesetzgebung 437-438.

${ }^{13}$ Verordnung des Reichsprotektors in Böhmen und Mähren vom 12. 12. 1940 über die Verlängerung und Abänderung einiger Bestimmungen des Verfassungsermächtigungsgesetzes vom 15.12. 1938 (VBl.RProt. S. 604).

${ }^{14}$ Dekret des Staatspräsidenten vom 8.3.1940 Nr. 83/1940 Slg., über den Amtseid der Regierungsmitglieder, der öffentlichen Angestellten und sonstiger Organe der öffentlichen Verwaltung.
}

und bei Gefahr im Verzug die im gemeinsamen Interesse notwendigen Anordnungen treffen. Die Verkündung von Gesetzen, Verordnungen und sonstigen Rechtsvorschriften sowie der Vollzug von Verwaltungsmaßnahmen und rechtskräftigen gerichtlichen Urteilen war auszusetzen, wenn der Reichsprotektor Einspruch einlegte. Die Verordnung über das Rechtssetzungsrecht im Protektorat Böhmen und Mähren vom 7. Juni 1939 (RGBl. I, S. 1039) erkannte dem Reichsprotektor das Recht zu, durch Verordnung das autonome Recht zu ändern, soweit das gemeinsame Interesse es erforderte. Bei Gefahr im Verzug konnte er Rechtsvorschriften jeder Art erlassen.

Die Rechtsvorschriften des Reichsprotektors, sowie alle Maßnahmen von Reichsbehörden waren einer Nachprüfung durch die Gerichte und Verwaltungsbehörden der autonomen Verwaltung entzogen.

Im Zusammenhang mit der Ernennung des neuen Reichsprotektors kam es im August 1943 zur Änderung seiner Stellung, Aufgaben und Kompetenzen. Die Vertretung der Interessen des Reiches im Protektorat ging auf das neu errichtete Deutsche Ministerium für Böhmen und Mähren über. ${ }^{15}$

Aus dem oben Erwähnten ist also klar ersichtlich, dass die Autonomie der Protektoratsregierung nur eine leere Phrase war, denn die legislative Tätigkeit der Regierung und der Ministerien des Protektorats unterstand der strengen Aufsicht des Amtes des Reichsprotektors. Die Ministerialbeamten arbeiteten oft unter der Aufsicht der Bevollmächtigten des Reichsprotektors und sie waren gezwungen, ihre Anweisungen zu befolgen. Das Aufsichtsrecht erstreckte sich auch auf den Erlass von Rechtsvorschriften durch autonome Organe auf Landes- und Be-

\footnotetext{
${ }^{15}$ Erlass des Führers über den Deutschen Staatsminister für Böhmen und Mähren vom 20.8.1943 (dRGB1. I, S. 527; zum Deutschen Staatsminister s. ausführlich KÜPPER, Karl Hermann Frank 31-52;
} 
zirksebene. In den ersten drei Jahren des Protektorats wurde das Aufsichtsrecht von außenstehenden deutschen Beamten durchgeführt, erst nach der Realisierung der Heydrich'schen Verwaltungsreform im Jahre 1942 kam es zur Besetzung der autonomen Behörden mit deutschen Beamten, wodurch nun direkt auf die Gesetzgebung Einfluss genommen werden konnte.

Im Februar 1942 wurde die Tätigkeit der Regierung als kollektives Organ eingestellt und ihre Zuständigkeit ging auf die einzelnen Minister über. ${ }^{16}$ Es waren nun zwei Typen von Verordnungen $\mathrm{zu}$ unterscheiden: Regierungsverordnungen und ministeriale Verordnungen. Die Verordnungen der Regierung des Protektorats mit Gesetzeskraft wurden, im Gegensatz zu früher, als die gesamte Regierung beteiligt war, künftig nur vom Vorsitzenden der Regierung und den mit ihrer Durchführung betrauten Ministern beschlossen und unterfertigt. Die anderen Ministerien nahmen am Gesetzgebungsprozess nur unter Ausübung ihres Rechts, Stellungnahmen zu den Entwürfen abzugeben, teil. Das den Entwurf einer Regierungsverordnung ausarbeitende Ministerium hatte die Zustimmung des Reichsprotektors einzuholen. Die ministerialen Verordnungen hatten die Minister zu erlassen, welche in der Regierungsverordnung mit der Durchführung betraut wurden.

Der letzte und schwerwiegendste Eingriff in die autonome Rechtsetzung wurde im Zusammenhang mit der Ausrufung des totalen Krieges im Sommer 1944 vorgenommen, als die fachlich zuständigen Minister ermächtigt wurden, „alle Verfügungen, die in ihrem Bereich zur Durchführung des totalen Kriegseinsatzes erforderlich waren und zu denen sonst ein Gesetz oder eine

\footnotetext{
${ }^{16}$ Verordnung des Reichsprotektors in Böhmen und Mähren vom 12. 12. 1940 über die Verlängerung und Abänderung einiger Bestimmungen des Verfassungsermächtigungsgesetzes vom 15.12. 1938 (VBl.RProt. S. 604).
}

Regierungsverordnung notwendig wäre, im Verordnungswege selbst zu treffen“. ${ }^{17}$

Zum Hauptverkündungsinstrument der autonomen Rechtssetzung gehörte die Sammlung der Gesetze und Verordnungen des Protektorats Böhmen und Mähren, wo Regierungsverordnungen, Verfassungsdekrete des Staatspräsidenten, ministeriale Verordnungen und Verordnungen allgemeinen Charakters der zentralen Behörden verkündet wurden. Bis zum 6. August 1940 erschien die Sammlung nur in tschechischer Sprache, danach zweisprachig. Der deutsche Text befand sich in der linken, der tschechische Text in der rechten Spalte. Da bis August 1940 keine offizielle Übersetzung des autonomen Rechts ins Deutsche zur Verfügung stand, wurde die deutsche Fassung der wichtigsten Rechtsvorschriften bis Ende 1942 in der Zeitschrift „Prager Archiv für Gesetzgebung und Rechtsprechung “18 veröffentlicht. $\mathrm{Zu}$ weiteren Verkündungsblättern des Protektorats gehörten: Das Amtsblatt des Protektorats Böhmen und Mähren, die Verordnungsblätter einzelner Ministerien, das Landesverordnungsblatt für Böhmen und das Landesverordnungsblatt für Mähren und Schlesien. Es erschienen nicht alle Verlautbarungsblätter zweisprachig.

\section{Die im Protektorat geltenden Reichsdeutschen Rechtsvor- schriften}

Wie schon oben angeführt, galten die Rechtsvorschriften des Reiches auf dem Protektoratsgebiet nicht einheitlich, sondern nur, wenn es sich aus ihrem Inhalt ergab oder wenn es in einer Rechtsvorschrift des Reiches ausdrücklich fest-

\footnotetext{
${ }^{17}$ Regierungsverordnung vom 22. 8. 1944 Nr. 177/1944 Slg., über die Ermächtigung der Minister zu außerordentlichen Verfügungen zur Durchführung des totalen Kriegseinsatzes.

${ }^{18}$ Husty, Prager Archiv.
} 
gesetzt wurde. ${ }^{19}$ Im Falle einiger Rechtsvorschriften war ihr Geltungsbereich schon aus dem Titel offensichtlich, oder in der Rechtsvorschrift wurde dem Geltungsbereich auf dem Protektoratsgebiet ein besonderer Teil gewidmet. Es gab jedoch auch Fälle, in denen aus der Formulierung hergeleitet werden musste, dass die Rechtsvorschrift für das gesamte Gebiet des Großdeutschen Reiches erlassen wurde. Es kam jedoch oft vor, dass nicht eindeutig ersichtlich war, ob und in welchem Umfang sich die reichsdeutsche Rechtsvorschrift auch auf das Gebiet des Protektorats bezog. Die in den tschechischen juristischen Fachzeitschriften veröffentlichten Kommentare beschrieben den Inhalt der reichsdeutschen Rechtsvorschriften mit einer Feststellung zum Schluss, dass „es nicht sicher sei, ob diese Rechtsnormen auch auf dem Gebiet des Protektorats Geltung haben."

Unklarheit herrschte auch darüber, ob sich die reichsdeutschen Rechtsnormen nur auf deutsche Staatsangehörige im Protektorat oder auch auf die tschechischen Staatsangehörigen des Protektorats beziehen sollten. Damit die tschechische juristische Fachöffentlichkeit überhaupt eine Übersicht über das im Protektorat geltende Reichsrecht hatte, wurden die Verzeichnisse der reichsdeutschen Vorschriften regelmäßig in tschechischen juristischen Fachzeitschriften abgedruckt sowie Übersichtssammlungen dieser Rechtsnormen herausgegeben. ${ }^{20}$

Der Geltungsbereich der Verkündungsblätter des Reichs erstreckte sich auch auf das Protektorat. Die Rechtsvorschriften des Reichs, die für das Protektorat Gültigkeit hatten und die in den Verkündungsblättern des Reichs verkündet werden, traten im Protektorat mit dem auf die

${ }^{19}$ Zum im Protektorat geltenden Reichsrecht s. näher SVATUŠKA, Říšskoněmecký právní řád 230.

${ }^{20}$ Z.B. VESELÝ, Říšské právo oder PUMPR, Rejstřík německých právních norem.
Verlautbarung in den Verkündungsblättern des Reichs folgenden Tag in Kraft. ${ }^{21}$

$\mathrm{Zu}$ den reichsdeutschen Verkündungsblättern mit Geltung für das Protektorat gehörten vor allem das Reichsgesetzblatt, der Deutsche Rechtsanzeiger und der Preußische Staatsanzeiger und die Ministerialblätter, wobei das Ministerialblatt des Reichs- und Preußischen Ministeriums des Innern das Wichtigste war. Da diese reichsdeutschen Verkündungsblätter selbstverständlich nur in deutscher Fassung herausgegeben wurden, stellte diese Tatsache ein relativ großes Hindernis dar, denn nicht alle Angehörige juristischer Berufe im Protektorat beherrschten das Juradeutsch auf einem so guten Niveau, dass sie den Wortlaut der reichsdeutschen Rechtsvorschriften verstanden.

Die breitere tschechische juristische Öffentlichkeit hatte oft nur einen beschränkten Zugang zu den reichsdeutschen Verkündungsblättern (vor allem zu ihren älteren Ausgaben), sodass nicht jeder die Möglichkeit hatte, sich mit dem Wortlaut der entsprechenden Rechtsnormen vertraut zu machen. Aus diesem Grund wurden ausgewählte reichsdeutsche Vorschriften mit Geltung für das Protektorat zusammen mit ihren offiziellen Übersetzungen ins Tschechische im Verordnungsblatt des Reichsprotektors abgedruckt. Die tschechischen offiziellen Übersetzungen wurden hier bis Ende 1941 abgedruckt, danach erfolgte keine Übersetzung mehr. Im Falle des Fehlens einer offiziellen Übersetzung in die tschechische Sprache wurden oft Privatübersetzungen angefertigt und in juristischen Fachzeitschriften oder Fachpublikationen veröffentlicht.

Ein Sonderamtsblatt für die Verkündung der reichsdeutschen Normen im Protektorat wurde schon mit der Bekanntmachung des Oberbefehlshabers des deutschen Heeres vom

${ }^{21}$ Verordnung des Reichsministers des Innern vom 3. 4.1939 über Rechtsvorschriften des Reichs für das Protektorat Böhmen und Mähren (dRGBl. I, S. 704). 
15. März 1939 errichtet. ${ }^{22}$ Im Verordnungsblatt für Böhmen und Mähren wurden Rechtsvorschriften und Anordnungen allgemeiner Art verkündet, die vom Oberbefehlshaber des Heeres in den unter den Schutz der deutschen Wehrmacht gestellten Gebieten getroffen wurden. Das Verordnungsblatt für Böhmen und Mähren gehörte auch zu den Verkündungsblättern des Reichs.

Im Zusammenhang mit der Beendigung der militärischen Verwaltung und mit dem Antritt des ersten Reichsprotektors wurde die Bezeichnung des Verkündungsblatt zu Verordnungsblatt des Reichsprotektors in Böhmen und Mähren geändert. ${ }^{23}$ Zwischen 1939 und 1941 erschien das Verordnungsblatt zweisprachig, in der linken Spalte befand sich die offizielle deutsche Fassung und in der rechten Spalte ihre tschechische Übersetzung. Seit 1942 wurde es nur auf Deutsch herausgegeben. Nach der Errichtung des Amtes des Deutschen Staatsministers für Böhmen und Mähren wurden, ab dem 20. August 1943, die Rechtsvorschriften im Verordnungsblatt des Deutschen Staatsministers für Böhmen und Mähren verkündet, welches das Verordnungsblatt des Reichsprotektors in Böhmen und Mähren ersetzte.

Wenn es aus Gründen der Dringlichkeit notwendig war, wurden die reichsdeutschen Rechtsnormen auch in der Tagespresse (in den Zeitungen „Der Neue Tag“ oder „Prager Abend"), ${ }^{24}$ durch öffentliches Aushängen ${ }^{25}$ oder im Rundfunk ${ }^{26}$ verkündet.

${ }^{22}$ Bekanntmachung des Oberbefehlshabers des Heeres vom 15.3. 1939 über das Verordnungsblatt für Böhmen und Mähren (VBl. S. 3).

${ }^{23}$ Diese Umbenennung wurde erst rückwirkend in der Verordnung über das Rechtsetzungsrecht im Protektorat Böhmen und Mähren vom 7.6.1939 (RGBl. I, S. 1039) verankert.

${ }^{24}$ Als Beispiel kann die Verordnung des Reichsprotektors vom 21.6.1939 über jüdisches Vermögen dienen, welche am Tage ihrer am 22.6. erfolgten Veröffentlichung in Prager Zeitung „Der Neue Tag“

\section{Fazit}

Das Grundproblem der im Protektorat Böhmen und Mähren geltenden Rechtsordnung bestand in ihrer Unübersichtlichkeit. Auf dem Gebiet Böhmens und Mährens galt eine Reihe von reichsdeutschen Rechtsnormen, die jedoch nicht nur im Reichsgesetzblatt, sondern auch in weiteren Verlautbarungsblättern des Reichs verkündet wurden, die im Protektorat schwieriger zugänglich waren. Das zeitgenössische Schrifttum wies auch immer wieder darauf hin, dass es sich aus den reichsdeutschen Rechtsvorschriften nicht ganz klar ergab, ob sich ihr Anwendungsbereich auch auf das Protektorat und gegebenenfalls auch auf die tschechische Bevölkerung bezog.

Im autonomen Recht oblag die rechtsetzende Gewalt dem Staatspräsidenten und der Protektoratsregierung, die zum "Treibzentrum" sämtlicher Legislative und Verwaltung wurde. ${ }^{27}$ Die Autonomie der Rechtsetzung wurde mit jedem weiteren Monat der Existenz des Protektorats ausgehöhlt und von Seiten des Amtes des Reichsprotektors und den Oberlandräten wurde in diese immer mehr eingegriffen.

In der Zeit des Protektorats erfuhren einige Rechtsgebiete keine wesentlicheren Änderungen, wie z. B. das Zivil- oder Handelsrecht, wäh-

in Wirksamkeit trat. Die Verkündung im Verordnungsblatt des Reichsprotektors erfolgte erst am 7. 7. 1939.

${ }^{25}$ Die Verkündung durch öffentliches Aushängen erfolgte z. B. im Falle der Verordnung des Reichsprotektors vom 26.8.1939 gegen Sabotagehandlungen (VB1.RPRot. S. 83).

${ }^{26}$ Z.B. wurde die Verordnung des Reichsministers der Luftfahrt vom 1.9. 1939 über das Verbot des gesamten Luftverkehrs über deutschem Hoheitsgebiet durch Rundfunk über den Deutschlandsender am 1. 9. 1939 um $4: 45$ Uhr verkündet und trat damit zu diesem Zeitpunkt in Wirksamkeit. Der Zeitpunkt des Inkraftretens wurde auch im Verordnungsblatt des Reichsprotektors (S. 104) veröffentlicht.

${ }^{27}$ HoffMANN, Nové zákony a nařízení 1942, 158. 
rend das Arbeitsrecht auf eine völlig neue Grundlage gestellt wurde. Während der Zeit der deutschen Besetzung in den böhmischen Ländern blieben die noch aus dem 19. Jahrhundert stammenden und nach 1918 aus der österreichischen Rechtsordnung übernommenen Gesetzbücher in Kraft und galten bis zu ihrer Aufhebung am Ende der vierziger Jahre. Es handelte sich um das Allgemeine Bürgerliche Gesetzbuch (1811), das Allgemeine Handelsgesetzbuch (1863), das Strafgesetzbuch (1852), die Zivilprozessordnung (1895) und die Strafprozessordnung (1873). Diese Gesetzbücher erfuhren nur geringfügige Änderungen und für die Durchführung wesentlicher Eingriffe in bestimmte Rechtsgebiete wurde die Form der selbstständig erlassenen Regierungsverordnungen gewählt. ${ }^{28}$

\section{Korrespondenz:}

Doc. JUDr. Jaromír TAUCHEN, Ph.D., LL.M.

Masaryk-Universität

Juristische Fakultät

Lehrstuhl für Staats- und Rechtsgeschichte

Veveří 70

CZ - 611 80, Brünn

jaromir.tauchen@law.muni.cz

ORCID-Nr. 0000-0001-9400-9484

\footnotetext{
${ }^{28}$ Aus dem deutschsprachigen Schrifttum zur Entwicklung der einzelnen Rechtsgebiete s. VOJÁČEK, SCHELLE, TAUCHEN, Die Entwicklung 119-124, 176180, 223-229, 255-259; TAUCHEN, Die Grundcharakteristik 56-60; DERS., Einige Bemerkungen 50-54; DERS., Die Anwendung 141-152.

$\mathrm{Zu}$ den konkreten Rechtsgebieten des auf dem Gebiet des Protektorats geltenden Rechts s. aus zeitgenössischem Schrifttum HOFFMANN, Nové zákony a nařízení 1939-1945; LORENZ, SCHINNERER, Das deutsche Strafrecht; VeSElÁ, Trestní právo; SOLNǍ̌, Trestní právo 93ff; SvatuŠKA, Právní vývoj 57-58.
}

\section{Abkürzungen:}

dRGB1. Deutsches Reichsgesetzblatt

Slg. Sammlung der Gesetze und Verordnungen der Tschechoslowakischen Republik/ des Protektorats Böhmen und Mähren

VB1. Verordnungsblatt für Böhmen und Mähren VB1.RProt.Verordnungsblatt des Reichsprotektors in Böhmen und Mähren

Siehe auch das allgemeine Abkürzungsverzeichnis: [http://www.rechtsgeschichte.at/media/abk.pdf]

\section{Literatur:}

Detlef BRANDES, Die Tschechen unter deutschem Protektorat, Bd. 1: Besatzungspolitik, Kollaboration und Widerstand im Protektorat Böhmen und Mähren bis Heydrichs Tod (1939-1942) (München-Wien 1969).

Josef Hoffmann (Hg.), Nové zákony a nařízení Protektorátu Čechy a Morava (Prag 1939-1945).

Franz Husty (Hg.), Prager Archiv für Gesetzgebung und Rechtsprechung (Prag 1939-1942).

Eva JANEČKOVÁ, Státoprávní uspořádání Protektorátu Čechy a Morava (1939-1945) (Pilsen 2013).

Helmut KRIESER (Hg.), Das neue Recht in Böhmen und Mähren (1.-3. Teil) (Prag 1943).

DERS., Die deutsche Gerichtsbarkeit im Protektorat Böhmen und Mähren. Ausübung und Umfang, in: Deutsches Recht, Ausgabe A, 10 (1940) 1745-1754.

René KüPPEL, Karl Hermann Frank als Deutscher Staatsminister für Böhmen und Mähren, in: Monika GLETTLER, Lubomír LIPTÁK, Alena MíšKOVÁ, Geteilt, besetzt, beherrscht: die Tschechoslowakei 1938-45: Reichsgau Sudetenland, Protektorat Böhmen und Mähren, Slowakei (Essen 2004) 3152.

Max LoRENZ, Erich SCHINNERER, Das deutsche Strafrecht im Reichsgau Sudetenland und im Protektorat Böhmen und Mähren (Prag 1940).

Pavel MARŠÁLEK, Pod ochranou hákového kříže: nacistický okupační režim v českých zemích 19391945 (Prag 2012).

Ders., Protektorát Čechy a Morava: státoprávní a politické aspekty nacistického okupačního režimu v českých zemích 1939-1945 (Prag 2002).

Tomáš PUMPR, Rejstř́k německých právních norem vydaných v době od 15 . března 1939 do 30. dubna 1940 s platností pro Čechy a Moravu a norem recipovaných (Prag 1939). 
Bernd RILL, Böhmen und Mähren. Geschichte im Herzen Mitteleuropas, Bd. II: Von der Romantik bis zur Gegenwart (Gernsbach 2006).

Karel SCHELLE, Jaromír TAUCHEN, Recht und Verwaltung im Protektorat Böhmen und Mähren (München 2009).

Vladimír SOLNǍ̌, Trestní právo německé a protektorátní, in: Právník 81 (1942) 93-100.

Ladislav SvATUŠKA, Řśšskoněmecký právní řád v Protektorátu Čechy a Morava, in: Moderní stát. Revue pro vědy státní 12 (1939) 225-231.

DERS., Právní vývoj v Protektorátu Čechy a Morava, in: Všehrd 21 (1940) 57-58.

František ŠTAJGR, Druhé nařízení o výkonu občanského soudnictví v Protektorátu, in: Všehrd 21 (1940) 177-181.

Jaromír TAUCHEN, Die Anwendung des deutschen Strafrechts im Protektorat Böhmen und Mähren, in: Publicationes Universitatis Miskolcinensis. Sectio Juridica et Politica 29 (2011) 141-152.

DERS., Die Grundcharakteristik des Privatrechts im Protektorat Böhmen und Mähren, in: Journal on European History of Law 2 (2011) 56-60.
DERS., Einige Bemerkungen zur Entwicklung des Arbeitsrechts im Protektorat Böhmen und Mähren, in: Journal on European History of Law 1 (2010) 50-54.

DERS., Ermächtigungsgesetzgebung in der Tschechoslowakei, in: Beiträge zur Rechtsgeschichte Österreichs 8 (2018) 428-440.

DERS., Vztah českého státu k Říši a jeho využití a zneužití v Protektorátu Čechy a Morava, in: Jaromír TAUCHEN, Karel Schelle (Hgg.), Státoprávní vztahy českého státu a římskoněmecké říše ve středověku a jejich důsledky pro další vývoj (Ostrava 2012) 106-120.

Jarmila VESELÁ, Trestní právo německé všeobecně platné na území Protektorátu Čechy-Morava (Prag 1939).

Vilém VeSELÝ, ̌̌íšské právo v Čechách a na Moravě: soubor říšských právních norem platných v Protektorátu Čechy a Morava a souvisejících a příbuzných predpisů protektorátních (Prag 19401943).

Ladislav VOJÁČEK, Karel SCHELLE, Jaromír TAUCHEN (Hgg.), Die Entwicklung des tschechischen Privatrechts (Brünn 2011). 